\title{
A crucial role for basic emotion awareness in the development of emotion regulation?
}

\author{
Alessandro Zuddas
}

Published online: 19 May 2012

(C) Springer-Verlag 2012

The regulation of emotions is a critical aspect of children's social and cognitive development. Over the course of development, the child learns to regulate actions that flow from negative emotion, such as hitting or yelling when angry and freezing or fleeing in the presence of fear. This happens first as a result of temperament and of a supportive caregiver environment and later as a function of voluntary or effortful control [1]. With the development of increasingly sophisticated regulatory abilities, children are able to regulate their actions more efficiently and more effectively in everyday emotional contexts.

Amygdala plays a crucial role in the generation of emotions: a large body of evidence suggests that amygdala activation to emotional faces increases between age 3 and 9 years [2], indicating that amygdala reactivity underlies an increasing sensitivity to emotionally relevant information. In turn, regions of the ventro-medial Prefrontal Cortex (vmPFC), especially the anterior cingulate cortex, have been implicated in the modulation of this emotional arousal. From a developmental perspective, the PFC as a whole is among the last brain areas to fully develop, with continued synaptogenesis and increasing white matter volume until late adolescence or early adulthood. This protracted developmental course has been linked to improvements in various complex cognitive skills, which are known to be important for the modulation of affective arousal [3, 4]. Effective connectivity between the anterior cingulate cortex and the

\footnotetext{
A. Zuddas $(\bowtie)$

Department of Biomedical Sciences, University of Cagliari,

Via Ospedale 119, 09124 Cagliari, Italy

e-mail: azuddas@unica.it

A. Zuddas

Unit of Child Neuropsychiatry, Cagliari University Hospital,

Cagliari, Italy
}

amygdala increases during periods of intense demand for emotion regulation and this effective connectivity increases with age [5].

A different approach to the study of emotion regulation is the detailed analysis of emotion awareness. In this issue of the Journal, the paper by Rieffe and de Rooij [6] investigates the longitudinal relation between emotion awareness and the development of internalizing symptoms.

Defining basic emotion as functional-response programmes finalized to quick adaptation to changes in the environment, the authors investigate whether the ability to recognize and manage emotions in childhood may modulate the development of internalizing symptoms (i.e. depression, fear, worry/rumination) in late childhood and early adolescence.

In a large sample of elementary school children (age 10 years on average), the authors administered a questionnaire designed with a six factor structure (6 subscales) measuring the ability to differentiate discrete emotions and locate the environmental changes that elicited the emotion (the "differentiation" scale), to talk about the emotion, to manage the bodily symptoms that are part of the emotion, the ability to communicate emotion (the "hiding emotion" scale), to analyse emotion and to attend to others emotion. They then related the questionnaire scores to the measures of depression, fears and worry/ruminations.

As previously shown, high scores in all six subscales predicted all the different internalizing symptoms, the impairment in understanding their own feelings contributed to symptoms of depression, high scores on the scale "hide emotion" predicted worrying and ruminative thoughts. After 2 years, an overall decline for the measure of depression, fears and worrisome thoughts was observed. However, this was the case for some but not all children. A sophisticate statistical analysis showed that the variance in emotion 
awareness was highly predictive for the trends for symptoms of depression, fear and worry/ruminative thoughts over time: the scale "differentiate emotions" predicted all the internalizing symptoms, whereas "attending to other emotions" and "analysis of emotion" contributed to the prediction of depressive symptoms, and "hiding emotions" contributed to the prediction of worry/rumination.

A recent line of evidence indicates the insula as a crucial area in emotion awareness. The insula is functionally connected with rostral-anterior cingulate cortex, the middle and inferior frontal cortex, and the temporo-parietal cortices, including amygdala. Functional maps of the human insula indicate that insular cortex may be differentiated in four functional regions defined by a sensorimotor, a cognitive, a social-emotional, and an olfactory-gustatory domain [7]. In good accordance with the anatomical findings in non-human primates, the anterior insula, in particular its anterior-basal part, is involved in the generation and mediation of feeling states as a response to environmental stimuli and affective states. In empathy-related processing, the insula is involved in the recognition of emotions in faces and the feeling of pain in others, processing emotions and emotion awareness in his anterior-basal part [7-10]. Although the insula developmental trajectory has been established [11], almost no study has investigated its role in the development of emotion regulation, or in the development of internalizing disorders. The results of the Rieffe and de Rooij papers strongly support a more intense research on emotion awareness and its underpinning brain circuitries.

The Chitsabesan et al. paper [12], in this issue, indirectly supports the need for further research on emotion awareness and recognition. The authors investigate the long term psychosocial and mental health needs of a group of juvenile offender (age 12-17) followed for 6 years. When interviewed as young adults, the majority of them continued to offend and were in custodial care or on a community order; two-third of the sample (54 male offenders) were in neither employment, nor in training. Almost 9 out 10 in the sample had a substance misuse disorder and a similar number met the criteria of antisocial personality disorder. A significant area of unmet need included problems with intimate relationship (i.e. difficulty in finding a partner or maintaining a close relationship). Interestingly, psychopathy scores measured by the Hare Psychopathy Checklist were not high, with impulsiveness, poor control behaviour and lacks of goals significantly more frequent than lack of remorse or lack of empathy.

The authors discuss the need for a personalized intervention for juvenile offenders suggesting the multidimensional family therapy (MDFT [13]) as one of the more cost-effective, evidence-based therapeutic approach.
In fact, emotion regulation, improving social competence and associated problem-solving skills are fundamental components of the MDFT. The poor behaviour control and lack of goals, together with the difficulties in finding a partner or maintaining a close relationship, strongly suggest a significant impairment in emotion awareness as a basic step of the emotion dysregulation in these young offenders.

Contrary to internalizing disorders, very few studies have accurately measured emotion awareness in conduct and other externalizing disorder: a putative role of the insula in these disorders has been almost completely ignored. A more intense investigation on the role of emotion awareness in the development of emotion regulation, and its neurobiological underpinning, could indeed open the way to innovative strategies in understanding developmental psychopathology and in designing more effective therapeutic approaches.

\section{References}

1. Calkins SD, Fox NA (2002) Self-regulatory processes in early personality development: a multilevel approach to the study of childhood social withdrawal and aggression. Dev Psychopathol 14:477-498

2. Todd RM, Evans JW, Morris D, Lewis MD, Taylor MJ (2011) The changing face of emotion: age-related patterns of amygdala activation to salient faces. Soc Cogn Affect Neurosci 6:12-23

3. Shaw P, Greenstein D, Lerch J, Clasen L, Lenroot R, Gogtay N, Evans A, Rapoport J, Giedd J (2006) Intellectual ability and cortical development in children and adolescents. Nature 440:676-679

4. Whittle S, Yap MB, Yücel M, Fornito A, Simmons JG, Barrett A, Sheeber L, Allen NB (2008) Prefrontal and amygdala volumes are related to adolescents' affective behaviors during parentadolescent interactions. Proc Natl Acad Sci USA 105:3652-3657

5. Perlman SB, Pelphrey KA (2012) Developing connections for affective regulation: age-related changes in emotional brain connectivity. J Exp Child Psychol 108:607-620

6. Rieffe C, de Rooij M (2012) The longitudinal relationship between emotion awareness and internalizing symptoms during late childhood. Eur Child Adolesc Psychiatry (this issue). doi: 10.1007/s00787-012-0267-8

7. Kurth F, Zilles K, Fox PT, Laird AR, Eickhoff SB (2010) A link between the systems: functional differentiation and integration within the human insula revealed by meta-analysis. Brain Struct Funct 214:519-534

8. Lamm C, Singer T (2010) The role of anterior insular cortex in social emotions. Brain Struct Funct 214:579-591

9. Craig AD (2010) The sentient self. Brain Struct Funct 214: 563-577

10. Cauda F, D'Agata F, Sacco K, Duca S, Geminiani G, Vercelli A (2011) Functional connectivity of the insula in the resting brain. NeuroImage 55:8-23

11. Shaw P, Kabani NJ, Lerch JP, Eckstrand K, Lenroot R, Gogtay N, Greenstein D, Clasen L, Evans A, Rapoport JL, Giedd JN, Wise SP (2008) Neurodevelopmental trajectories of the human cerebral cortex. J Neurosci 28:3586-3594 
12. Chitsabesan P, Rothwell J, Kenning C, Law H, Carter LA, Bailey S, Clark A (2012) Six years on: a prospective cohort study of male juvenile offenders in secure care. Eur Child Adolesc Psychiatry (this issue). doi:10.1007/s00787-012-0266-9
13. Liddle HA, Dakof GA, Turner RM, Henderson CE, Greenbaum PE (2008) Treating adolescent drug abuse: a randomized trial comparing multidimensional family therapy and cognitive behavior therapy. Addiction 103:1660-1670 